EPJ Web of Conferences 40, 16007 (2013)

DOI: $10.1051 /$ epjconf/20134016007

(c) Owned by the authors, published by EDP Sciences, 2013

\title{
Programmable Input for Nanomagnetic Logic Devices
}

\author{
J. Kiermaier ${ }^{1, a}$, S. Breitkreutz ${ }^{1}$, I. Eichwald ${ }^{1}$, X. Ju ${ }^{2}$, G. Csaba ${ }^{3}$, D. Schmitt-Landsiedel ${ }^{1}$, and M. Becherer ${ }^{1}$ \\ 1 Lehrstuhl für Technische Elektronik, Technische Universität München, Theresienstraße 90, 80333 Munich, Germany. \\ 2 Lehrstuhl für Nanoelektronik, Technische Universität München, Theresienstraße 90, 80333 Munich, Germany. \\ 3 Center for Nano Science and Technology, University of Notre Dame, Notre Dame, IN 46556, USA.
}

\begin{abstract}
A programmable magnetic input, based on the magnetic interaction of a soft and hard magnetic layer is presented for the first time. Therefore, a single-domain $\mathrm{Co} / \mathrm{Pt}$ nanomagnet is placed on top of one end of a permalloy bar, separated by a thin dielectric layer. The permalloy bar of the introduced input structure is magnetized by weak easy-axis in-plane fields. Acting like a 'magnetic amplifier', the generated fringing fields of the permalloy pole are strong enough to control the magnetization of the superimposed $\mathrm{Co} / \mathrm{Pt}$ nanomagnets, which have high crystalline perpendicular magnetic anisotropy. This magnetostatic interaction results in a shift of the hysteresis curve of the $\mathrm{Co} / \mathrm{Pt}$ nanomagnet, measured by magneto-optical Kerr microscopy. The Co/Pt nanomagnet is fixed by the fringing field of the permalloy and thereby not affected by the magnetic power clock of the Nanomagnetic Logic system. MFM measurements verify the functionality of the programmable magnetic input structure. The fringing fields are extracted from micromagnetic simulations and are in good agreement with experimental results. The introduced input structure enables switching the logic functionality of the majority gate from NAND to NOR during runtime, offering programmable Nanomagnetic Logic.
\end{abstract}

\section{Introduction}

Nanomagnetic Logic (NML) is envisioned to complement state-of-the-art CMOS technology by providing non-volatile computing states for low power applications [1]. Basic elements of these systems are ferromagnetic, singledomain nanomagnets with either in-plane [2] or perpendicular magnetization [3] [4]. In this work, Co/Pt multilayer magnets with perpendicular crystalline anisotropy are favored, due to their higher thermal stability and larger design space in shape and switching field modification by focused ion beam (FIB) irradiation [5].

Fig. 1 demonstrates the assembly of a nanomagnetic computing system. The bistable magnets interact with their next neighbors by magnetic field-coupling. The information is transmitted between the logic elements by magnetic inverter chains [6], in which well-defined local FIB irradiation is used to generate artificial nucleation centers. These weakest links govern the reversal process of the magnets and thereby ensure the directed signal flow. Logic functionality is implemented in the system by majority gates, as illustrated in Fig 1. The computing dot $\mathrm{C}$ perceives the fringing fields of its three left input neighbors $D_{1}, D_{2}$ and $P$. The superposition of these magnetic fields forces the magnetization of the computing dot in antiparallel direction of the majority of its input magnets[7]. Due to the strong crystalline anisotropy, an additional magnetic clocking field is necessary to overcome the switching threshold of the magnets and to enable the fringing fields to govern the magnetization direction of their following magnets.

The complex logic functionality of the majority gate can be constricted to NAND or NOR by implementing one input with fixed magnetization. This enables the exploitation of standard CMOS design tools for NML. The coer-

a e-mail: kiermaier@tum.de

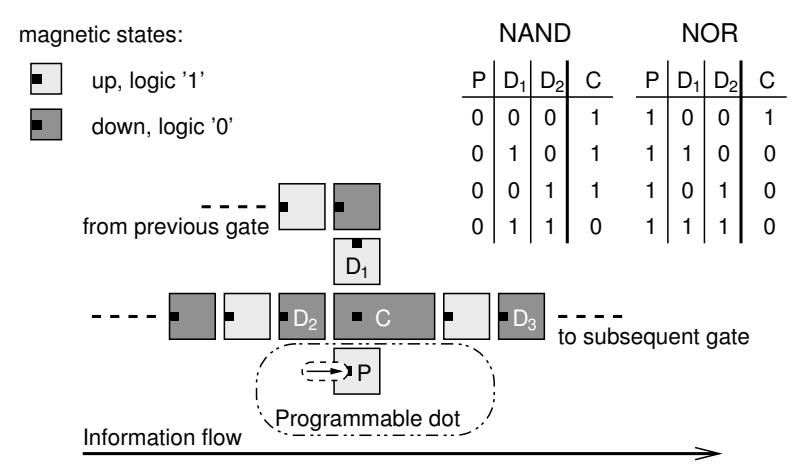

Fig. 1. Design of a field-coupled Nanomagnetic Logic device. The logic functionality of the majority gate is selectable by the programmable input.

civity of this programmable magnet must be sufficiently high, that its magnetization state is not affected by the magnetic power clock. By integrating a feature, that enables controlled switching of the programmable dot during runtime without influencing any other logic component, NML can be expanded to reconfigurable logic.

Investigations on the interaction between magnetic layers offer new opportunities for NML devices [8]. In this work, this magnetic interaction is used to experimentally demonstrate a programmable hard-magnetic nanodot suitable for on-chip programmable magnetic inputs in NML systems.

\section{Theory}

As all magnets of the envisioned NML system are patterned from the same magnetic thin film, they have about 
a)

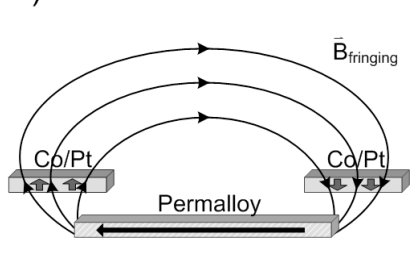

b)

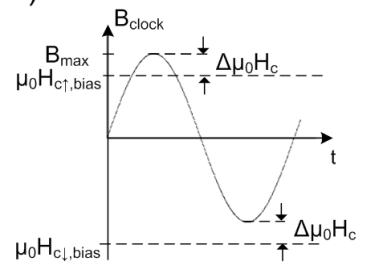

a)

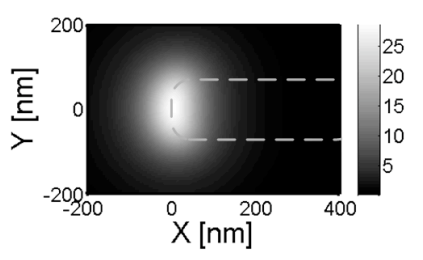

b)

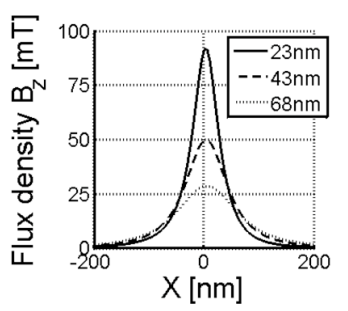

Fig. 2. a) Schematic of the magnetic input device. b) Characteristics of the sinusoidal magnetic power clock. The biasing field, generated by the permalloy pole is supporting the up-switching of the $\mathrm{Co} / \mathrm{Pt}$ magnet by $\Delta \mu_{0} \mathrm{H}_{\mathrm{c}}$. The biased coercivities $\mu_{0} \mathrm{H}_{\mathrm{c} \uparrow \text {,bias }}$ and $\mu_{0} \mathrm{H}_{\mathrm{c} \downarrow \text {,bias }}$ of the $\mathrm{Co} / \mathrm{Pt}$ magnet are marked.

the same switching fields, neglecting fabrication variations and thermal fluctuations [9] [10]. The fabrication of a magnet with higher coercivity can be realized by using a lower ion dose when the artificial nucleation center is created by FIB irradiation [11]. This harder magnet must be programmed at the beginning of operation of the system and its magnetization state can not be changed during runtime. In this paper, we present an advanced method to create a hard magnetic dot that allows to be programmed during runtime.

Fig 2a) shows a schematic of the investigated input structure. A permalloy bar is located below the layer with $\mathrm{Co} / \mathrm{Pt}$ magnets. The perpendicular component of the fringing fields of the permalloy pole $\mathrm{B}_{\text {fringing }}$ act on the $\mathrm{Co} / \mathrm{Pt}$ magnet, especially in the artificial nucleation center and thereby support or prevent its switching. The direction of the fringing field is conditioned by the magnetization direction of the permalloy bar. It can be programmed by a magnetic field pulse in in-plane direction, which is the hard-magnetic axis of the $\mathrm{Co} / \mathrm{Pt}$ and is therefore not influencing the magnetization states of the $\mathrm{Co} / \mathrm{Pt}$ logic. Due to a reduced switching threshold of $\mathrm{Co} / \mathrm{Pt}$ magnets by applying in-plane magnetic fields, it is beneficial to synchronize the magnetic power clock and the programming in-plane pulse by applying it at almost zero out-of-plane magnetic field.

The switching field of the permalloy bar is lower than the coercivity of the $\mathrm{Co} / \mathrm{Pt}$ magnets due to pure shape anisotropy. This enables low power programming of the magnet and reconfiguration of the magnetization state of the programmable $\mathrm{Co} / \mathrm{Pt}$ dot during runtime. The permalloy bar behaves like a 'magnetic amplifier', which is controlled by weak magnetic field pulses and provides strong fringing fields for the $\mathrm{Co} / \mathrm{Pt}$ magnet.

The effect of the fringing field of the permalloy pole on the $\mathrm{Co} / \mathrm{Pt}$ magnet is visualized in Fig 2b). NML systems are clocked by a sinusoidal magnetic power clock with a maximum flux density $B_{\max }$ equal to the coercivity of the magnets. This power clock is necessary to overcome the switching threshold of the $\mathrm{Co} / \mathrm{Pt}$ magnets. At maximum flux density, the $\mathrm{Co} / \mathrm{Pt}$ magnets are in metastable state and the coupling fields from neighboring dots govern their magnetization state, enabling logic computation and information transport. The fringing field of the permalloy pole acts continuously on the input $\mathrm{Co} / \mathrm{Pt}$ magnet. This magnetic bias is shifting the hysteresis curve of the $\mathrm{Co} / \mathrm{Pt}$ input magnet by $\Delta \mu_{0} \mathrm{H}_{\mathrm{c}}$. It results in an asymmetric hysteresis curve with one-side increased coercivity $\mu_{0} \mathrm{H}_{\mathrm{c} \downarrow \text {,bias }}$,
Fig. 3. a) OOMMF simulation of the perpendicular magnetic flux density $B_{z}[\mathrm{mT}]$, generated by a permalloy pole. The simulated plane is $68 \mathrm{~nm}$ above the pole. The contour of the end of the permalloy bar is visualized as gray dashed line. b) OOMMF simulation of $B_{z}$ of the permalloy pole at different distances above the pole.

which will not be overcome by the magnetic power clock. Thereby, the magnetization state of the $\mathrm{Co} / \mathrm{Pt}$ input magnet is fixed as long as the magnetization state of the permalloy bar is in a steady state.

The fringing fields of a permalloy bar bias a $\mathrm{Co} / \mathrm{Pt}$ magnet in the layer above and thereby govern its magnetization state during magnetic clocking. The biased $\mathrm{Co} / \mathrm{Pt}$ magnet fulfills all demands for a programmable input in NML devices. The following modification extends the range of applications. By fabricating the permalloy bars with their magnetic easy-axis in different directions, the magnets can be programmed separately. Areas on a chip can be united by identical orientation of the permalloy bars. The functionality of those logic blocks can be switched by applying an aligned in-plane field pulse, without influencing other blocks with tilted easy-axis direction.

\section{Simulation}

Micromagnetic simulations with OOMMF are performed on the proposed programmable input. The fringing fields of a $20 \mathrm{~nm}$ thick permalloy bar are simulated to estimate the interaction with the $\mathrm{Co} / \mathrm{Pt}$. The size of the permalloy bar is $140 \mathrm{~nm}$ by $2.1 \mu \mathrm{m}$ to ensure single domain state. Note, both poles of the permalloy bar may be used for the $\mathrm{Co} / \mathrm{Pt}$ logic. In the simulation, the permalloy bar is magnetized in easy-axis direction and subsequently relaxed before the designated data is extracted.

Fig 3a) shows the perpendicular magnetic flux density in a plane $68 \mathrm{~nm}$ above the permalloy bar, where the $\mathrm{Co} / \mathrm{Pt}$ magnets are located in the experiment. The contour of the permalloy is marked by the dashed line. Micromagnetic simulations showed, that rounded edges are focusing the fringing field in the center of the end of the bar. Fig $3 b$ ) shows the simulated perpendicular flux density, generated by the permalloy pole at the center line $y=0 \mathrm{~nm}$ at different planes above the pole. Increasing the distance from $23 \mathrm{~nm}$ to $68 \mathrm{~nm}$ reduces the maximum flux density clearly from $91 \mathrm{mT}$ to $29 \mathrm{mT}$. By increasing the width of the permalloy bar, the width of the pole increases simultaneously with almost constant maximum flux density. Simulations on a $250 \mathrm{~nm}$ long bar verified, that reducing the length does not affect the amplitude of the fringing field as long as the shape anisotropy guarantees the poles on the 
a)

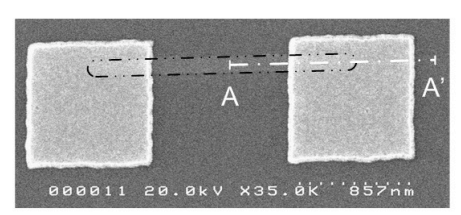

b)

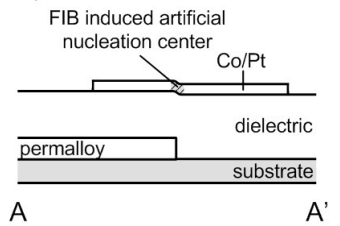

Fig. 4. a) SEM image of the fabricated sample. The buried and planarized permalloy bar is framed by a dashed line. b) Schematic cross-section of the structure, illustrating the configuration of the permalloy bar and the $\mathrm{Co} / \mathrm{Pt}$ nanomagnet. Its artificial nucleation center is created on top of the permalloy pole.

left and right end of the bar. Enlarging the thickness of the permalloy bar increases the fringing fields almost linearly.

Micromagnetic simulations including the permalloy bar and a $\mathrm{Co} / \mathrm{Pt}$ magnet visualized negligible reverse interaction from the $\mathrm{Co} / \mathrm{Pt}$ back to the permalloy if both magnetic layers are separated by a dielectric layer. These forces are considerably weak and thereby not affecting the magnetization state of the permalloy bar. In contrast, the micromagnetic simulation showed a fringing field of the permalloy bar, that is strong enough to control the magnetization of the superimposed $\mathrm{Co} / \mathrm{Pt}$ nanomagnet.

\section{Experiment}

\subsection{Sample fabrication}

$\mathrm{Co} / \mathrm{Pt}$ nanomagnets are placed on top of one end of a permalloy bar, separated by a thin dielectric layer. The $20 \mathrm{~nm}$ thick permalloy is evaporated on a silicon substrate, using a $2 \mathrm{~nm}$ Ti adhesion layer and it is structured in a lift-off process. $75 \mathrm{~nm}$ of hydrogen silsesquioxanes (HSQ) are spin coated as dielectric interlayer to adjust the distance of the permalloy pole to the $\mathrm{Co} / \mathrm{Pt}$ nanomagnet in the way of planarizing the permalloy bar and to provide a smooth surface for the overlying Co/Pt layer. The $\mathrm{Ti}_{1 \mathrm{~nm}} \mathrm{Pt}_{5 \mathrm{~nm}} /\left[\mathrm{Co}_{0.6 \mathrm{~nm}}+\right.$ $\left.\mathrm{Pt}_{1.0 \mathrm{~nm}}\right]_{3} / \mathrm{Pt}_{3 \mathrm{~nm}}$ multilayer stack is magnetron sputtered at room temperature. A $4 \mathrm{~nm}$ Ti layer is evaporated and structured in a lift-off process, serving as hard mask in the dry-etching process. The $\mathrm{Co} / \mathrm{Pt}$ nanomagnets are patterned by Ar-ion beam etching stopping in the HSQ layer. The artificial nucleation center in the $\mathrm{Co} / \mathrm{Pt}$ nanomagnet is generated by local FIB irradiation at a dose of $1 \cdot 10^{14} \mathrm{ions} / \mathrm{cm}^{2}$, using a Micrion 9500 system with a gallium source and an acceleration voltage of $50 \mathrm{kV}$. The size of the artificial nucleation center is $50 \mathrm{~nm}$ by $50 \mathrm{~nm}$.

Fig 4a) shows an SEM image of the fabricated structure. The $1 \mu \mathrm{m}$ by $1 \mu \mathrm{m} \mathrm{Co} / \mathrm{Pt}$ nanomagnets are clearly visible. The $140 \mathrm{~nm}$ by $2.1 \mu \mathrm{m}$ permalloy bar with rounded edges, framed by the dashed line, provides a weak contrast to the surrounding, because it is buried under the HSQ layer. Fig 4b) visualizes a schematic cross-section of the fabricated programmable input structure. Atomic force microscopy illustrates, that the HSQ planarizes the sharp $20 \mathrm{~nm}$ thick edge of the permalloy bar to a $7 \mathrm{~nm}$ smooth passage for the $\mathrm{Co} / \mathrm{Pt}$ film. The distance of the center of the $\mathrm{Co} / \mathrm{Pt}$ magnet and the top of the permalloy bar is calculated to be $68 \mathrm{~nm}$, as used in the micromagnetic simulation. Due to the concentrated fringing fields of the permalloy bar a)

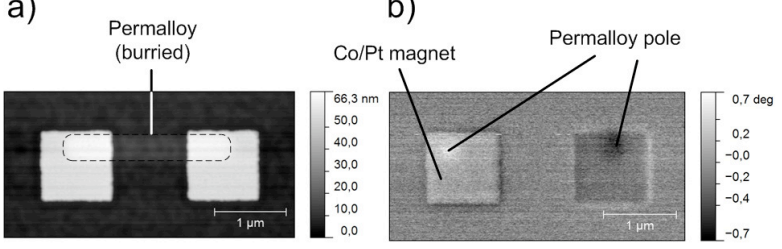

Fig. 5. MFM image of the programmable input structure. a) In the topography the subjacent permalloy wire is schematically visualized. b) The MFM phase image shows the magnetization of $\mathrm{Co} / \mathrm{Pt}$ nanomagnets, controlled by the permalloy poles.

in a small area (see Fig 3) the adjustment of the artificial nucleation center on top of the permalloy pole is the vital step in the fabrication process.

\subsection{Magnetic force microscopy}

Magnetic force microscopy (MFM) is performed to visualize the magnetization orientation of the permalloy and the $\mathrm{Co} / \mathrm{Pt}$. First, the permalloy bar is magnetized by a $300 \mathrm{mT}$ in-plane field to definitely align the magnetization in easyaxis. Afterwards a decreasing oscillating out-of-plane magnetic field with max amplitude of $120 \mathrm{mT}$ is used to drive the $\mathrm{Co} / \mathrm{Pt}$ nanomagnets to ground state.

Fig 5a) shows the MFM topography image of the programmable input structure. The $\mathrm{Co} / \mathrm{Pt}$ nanomagnets are clearly visible as bright, square areas. Due to the not complete planarization of the HSQ, the buried permalloy bar is also visible in the topography. Fig 3b) shows the MFM phase image. The fringing fields of the permalloy poles are visible as bright and dark spot at the ends of the permalloy bar. Their size is in good agreement with the simulation in Fig 5a). The $\mathrm{Co} / \mathrm{Pt}$ magnets appear bright and dark in the image, representing their perpendicular magnetization state. During driving the $\mathrm{Co} / \mathrm{Pt}$ into ground state, the fringing field of the permalloy acts as bias field and thereby governs the magnetization direction of the $\mathrm{Co} / \mathrm{Pt}$ magnet in ground state. In the MFM measurement, the direction of the fringing fields of the permalloy corresponds to the magnetization of the $\mathrm{Co} / \mathrm{Pt}$ magnets, verifying the functionality of the fabricated programmable input device.

\subsection{Hysteresis curve measurements}

Hysteresis curve measurements are used to quantify the interaction between the two magnetic layers. A magneto optical Kerr microscope (MOKE) is employed for fast and contactless hysteresis measurements. In the experiment, the permalloy bar is magnetized by an in-plane magnetic field pulse. Subsequently, the hysteresis curves of the $\mathrm{Co} / \mathrm{Pt}$ nanomagnets on top of both poles of the permalloy bar are measured by applying an out-of-plane field with maximum amplitude of $150 \mathrm{mT}$. Afterwards, the magnetization of the permalloy is reversed and the hysteresis measurements of the $\mathrm{Co} / \mathrm{Pt}$ magnets are repeated.

In Fig 6 the distribution of the coercivity of 30 hysteresis curves of one $\mathrm{Co} / \mathrm{Pt}$ magnet is plotted for both magnetization states of the permalloy. Regarding the black distributions, the fringing fields of the permalloy pole are oriented out of the plane. This bias field supports the switching of the $\mathrm{Co} / \mathrm{Pt}$ magnet at positive external magnetic fields 


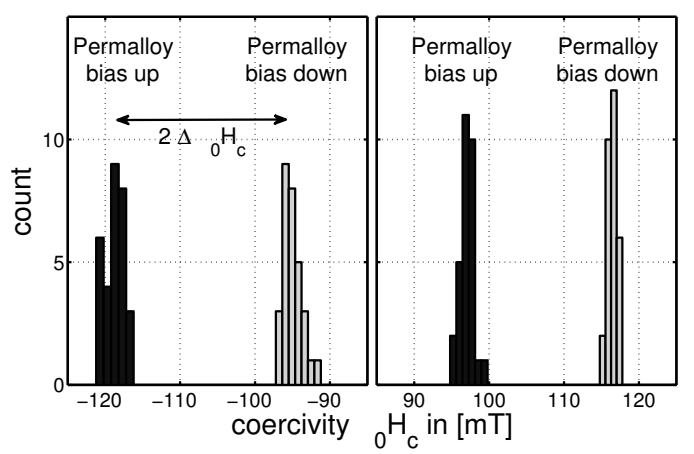

Fig. 6. Coercivity measurements by MOKE show a shift $\Delta H_{c}$ in the hysteresis curve of the $\mathrm{Co} / \mathrm{Pt}$ nanomagnet, depending on the bias field generated by the permalloy pole.

and counteracts the switching at negative external magnetic fields. The resulting hysteresis curve of the $\mathrm{Co} / \mathrm{Pt}$ is shifted to negative fields. The gray distributions show the shift to positive fields at reversed permalloy magnetization. The extracted mean shift of the coercivity is $2 \Delta \mu_{0} \mathrm{H}_{\mathrm{c}}=$ $21.3 \mathrm{mT}$. Thus, the original hysteresis curve of the $\mathrm{Co} / \mathrm{Pt}$ is shifted by the magnetostatic interaction, generated by the permalloy pole by $\pm 10.6 \mathrm{mT}$. The $\mathrm{Co} / \mathrm{Pt}$ magnet on the other side of the permalloy bar shows a shift in opposite direction with same $\Delta \mu_{0} \mathrm{H}_{\mathrm{c}}$, due to the opposite direction of the fringing fields, generated by the other permalloy pole. The switching field distribution of the $\mathrm{Co} / \mathrm{Pt}$ magnet in each magnetization state is thermally induced [10].

The OOMMF simulation predicted a maximum amplitude of the fringing field of $29 \mathrm{mT}$, which is larger than the measured value of $10.6 \mathrm{mT}$ in the experiment. The reason is an inaccuracy in the alignment of the artificial nucleation center, which was visible in the image, recorded during the FIB irradiation process. Regarding Fig 3, already an offset of $60 \mathrm{~nm}$ in both axis reduces the amplitude of the fringing field at the artificial nucleation center from $29 \mathrm{mT}$ to $10.6 \mathrm{mT}$. This emphasizes the accurate alignment of the artificial nucleation center to maximize the interaction between both magnetic layers.

After the hysteresis curve measurements with maximal out-of-plane magnetic flux densities of $150 \mathrm{mT}$, MFM measurements prove, that the in-plane magnetization state of the permalloy was not affected by the applied magnetic fields, demonstrating the robustness of the investigated device. The measured magnetostatic interaction of the permalloy bar on the $\mathrm{Co} / \mathrm{Pt}$ magnet is sufficiently strong to implement a programmable input magnet in NML using the proposed concept.

\section{Conclusion}

The magnetic interaction between a permalloy magnet and a $\mathrm{Co} / \mathrm{Pt}$ magnet is exploited to realize a programmable input for Nanomagnetic Logic with perpendicular magnetic anisotropy. The fringing fields of the patterned permalloy act like a 'magnetic amplifier', providing a local magnetic bias field and thereby control the magnetization state of the $\mathrm{Co} / \mathrm{Pt}$ magnet. The interaction is estimated by micromagnetic simulations, predicting a maximum coupling flux density of $29 \mathrm{mT}$ for the investigated permalloy magnet in a locally confined area at a distance of $68 \mathrm{~nm}$ above the permalloy bar. Simulations also confirm stronger interaction at reduced distance between the magnetic layers and at a thicker permalloy layer. Magnetic force microscopy measurements verified the functionality of the proposed programmable input device. The magnetization of the $\mathrm{Co} / \mathrm{Pt}$ magnets in ground state appeared correctly oriented by the fringing fields of the permalloy poles in the MFM image. Hysteresis curve measurements quantify the magnetic bias field of the permalloy pole at the $\mathrm{Co} / \mathrm{Pt}$ magnet. Its coercivity is shifted by $\Delta \mu_{0} \mathrm{H}_{\mathrm{c}}= \pm 10.6 \mathrm{mT}$, depending on the orientation of the fringing fields. The measured value corresponds to the micromagnetic simulation, if an inaccuracy in the alignment of the artificial nucleation center of the $\mathrm{Co} / \mathrm{Pt}$ magnet is considered. This emphasizes the necessity of the accurate alignment of the artificial nucleation center for maximum interaction. The introduced magnetic input structure is used to switch the logic functionality of majority gates during runtime and envisions programmable Nanomagnetic Logic.

\section{Acknowledgment}

The authors would like to thank S. Boche for assistance in sample fabrication and F. Limbrunner for preparative experiments. We also thank the DFG (Grant SCHM 1478/91 and Grant CS 62/2-1) and the Technische Universität München - Institute for Advanced Study, funded by the German Excellence Initiative, for financial support.

\section{References}

1. The International Technology Roadmap for Semiconductors (ITRS): Emerging Research Devices (ERD), http://www.itrs.net (2011)

2. A. Imre, G. Csaba, L. Ji, A. Orlov, G. Bernstein, W. Porod, Science 311, 205 (2006)

3. M. Becherer, G. Csaba, R. Emling, W. Porod, P. Lugli, D. Schmitt-Landsiedel, in ISSCC (2009), pp. 474-475

4. G. Csaba, P. Lugli, M. Becherer, D. SchmittLandsiedel, W. Porod, Journal of Computational Electronics 7, 454 (2008)

5. S. Breitkreutz, J. Kiermaier, S.V. Karthik, G. Csaba, D. Schmitt-Landsiedel, M. Becherer, Journal of Applied Physics 111, A715 (2012)

6. I. Eichwald, A. Bartel, J. Kiermaier, S. Breitkreutz, G. Csaba, D. Schmitt-Landsiedel, M. Becherer, IEEE Transactions on Magnetics, to be published (2012)

7. S. Breitkreutz, J. Kiermaier, I. Eichwald, X. Ju, G. Csaba, D. Schmitt-Landsiedel, M. Becherer, IEEE Transactions on Magnetics, to be published (2012)

8. G. Csaba, J. Kiermaier, M. Becherer, S. Breitkreutz, X. Ju, P. Lugli, D. Schmitt-Landsiedel, W. Porod, Journal of Applied Physics 111, E337 (2012)

9. T. Thomson, G. Hu, B.D. Terris, Physical Review Letters 96 (2006)

10. J.B.C. Engelen, M. Delalande, A..J. le Fèbre, T. Bolhuis, T. Shimatsu, N. Kikuchi, L. Abelmann, J.C. Lodder, Nanotechnology 21 (2010)

11. J.H. Franken, M. Hoeijmakers, R. Lavrijsen, H.J.M. Swagten, J. Phys.: Condens. Matter 24, 024216 (2012) 